\title{
Maladie de Sutton : difficultés thérapeutiques. A propos d'une observation clinique
}

\section{Sutton's disease: therapeutic difficulty. A case report}

HASSAN BAYI ${ }^{1}$, BOUCHRA TALEB ${ }^{2}$, WAFAA EL WADY²

\begin{abstract}
RÉSUMÉ
La maladie de Sutton, appelée aussi periadenitis mucosa necrotica reccurens, représente approximativement $10 \%$ de l'ensemble des aphtoses buccales récidivantes. Les lésions mesurent plus de $1 \mathrm{~cm}$ de diamètre, évoluent pendant des semaines, laissent des cicatrices rétractiles.

On rapporte un cas de maladie de Sutton où plusieurs traitements ont été essayés sans pouvoir prévenir les récidives. Néanmoins, en l'absence de traitement capable de prévenir la récidive des ulcérations, il existe plusieurs modalités thérapeutiques susceptibles de réduire la fréquence et la sévérité de ces lésions. Med Buccale Chir Buccale 2007; $13: 41-45$.
\end{abstract}

mots clés: maladie de Sutton, périadénite de Sutton, traitement.

médecine

buccale

chirurgie

\section{SUMMARY}

buccale

Introduction. Sutton's disease, also known a periadenitis mucosa necrotica reccurens, occurs in approximately $10 \%$ of recurrent aphtous ulcers patients. The lesions are exceed $1 \mathrm{~cm}$ in diameter, persist for up to 6 week, and often heal with scarring.

We report one case of Sutton's disease successfully treated by combined modality treatment. There is no curative therapy to prevent the recurrence of ulcers, and all available treatment modalities can reduce the frequency or severity of the lesions. Med Buccale Chir Buccale 2007; 13 : 41-45.

key words: Sutton's disease, periadenitis of Sutton, treatment. 
médecine buccale chirurgie buccale

VOL. $14, \mathrm{~N}^{\circ} 1$ 2008

page 42
La maladie de Sutton, appelée aussi aphte cicatriciel majeur, stomatite nécrosante, aphtose géante, aphte nécrosant, périadénite de Sutton ou encore periadenitis mucosa necrotica reccurens, est une aphtose buccale décrite comme la forme majeure de l'aphtose buccale récidivante. Cette lésion a été décrite pour la première fois en 1911 par Richard L. Sutton, dermatologue américain, ayant observé une lésion inflammatoire de la muqueuse buccale se développant autour des glandes salivaires accessoires ${ }^{[1,2]}$.

La maladie se caractérise par une ou plusieurs ulcérations (aphtes) de taille importante, qui persistent pendant plusieurs semaines ${ }^{[1]}$. Les ulcérations sont larges et profondes. La guérison des lésions laisse une cicatrice rétractile. L'attitude thérapeutique repose sur des protocoles locaux et généraux dont l'efficacité reste limitée.

Cet écrit décrit le cas d'un enfant de 13 ans atteint d'une maladie de Sutton, où des traitements multiples ont été essayés sans véritable efficacité.

\section{OBSERVATION}

B. J., âgé de 11 ans, physiquement très chétif, vient consulter pour des cicatrices rétractiles sur la face interne des joues qui ont entraîné une limitation de l'ouverture buccale. L'anamnèse ne retrouve aucun antécédent médico-chirurgical. Le patient était suivi dans un dispensaire pour des ulcérations buccales, traitées par antibiothérapie et l'application locale d'antiseptique.

Le patient, anxieux, rapporte des douleurs intenses à type de brûlures, associées à une dysphagie. L'examen des aires ganglionnaires montrait une polyadénopathie sub-mandibulaire. A l'examen endobuccal, on note une mauvaise hygiène buccodentaire et des brides rétractiles dans chaque joue, plus marquées dans les régions rétro-commissurales, apparues après la guérison de lésions très douloureuses, ayant évolué en moyenne 20 jours, avant de cicatriser spontanément (Fig. 1). Lors d'une autre consultation, on a constaté la présence d'une ulcération de $13 \mathrm{~mm}$, douloureuse, siégeant sur la lèvre inférieure sur un site cicatriciel ; la lésion était ovalaire, à bords déchiquetés, reposant sur un nodule inflammatoire légèrement saillant, avec un

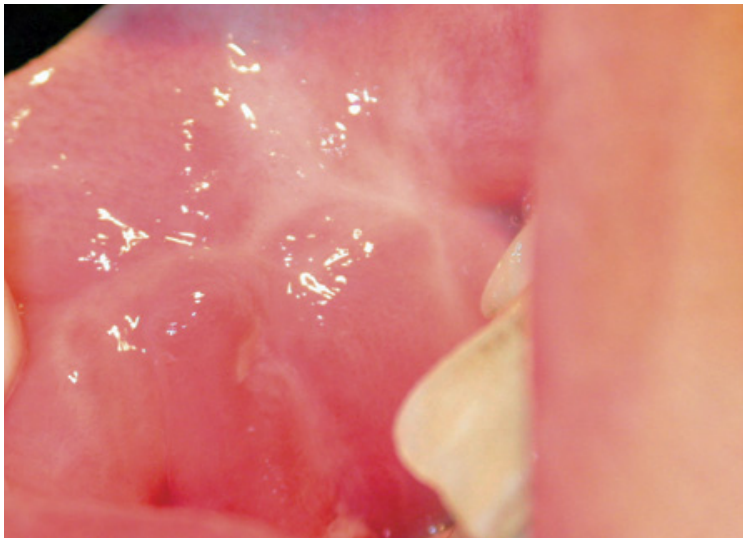

Figure 1 : Brides cicatricielles rétractiles rétro-commissurales droites.

Right sided retrocommissural post healing scars.

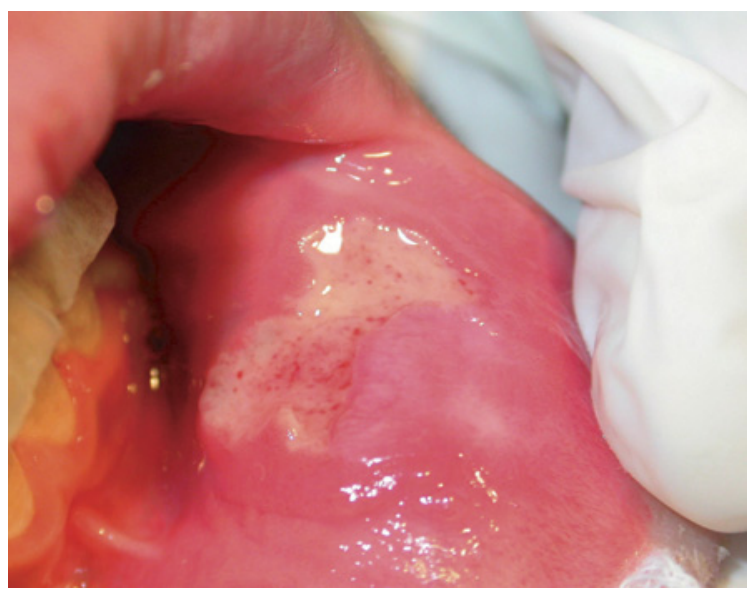

Figure 2 : Large ulcération siégeant sur la lèvre inférieure.

A large size ulcer on the lower lip.

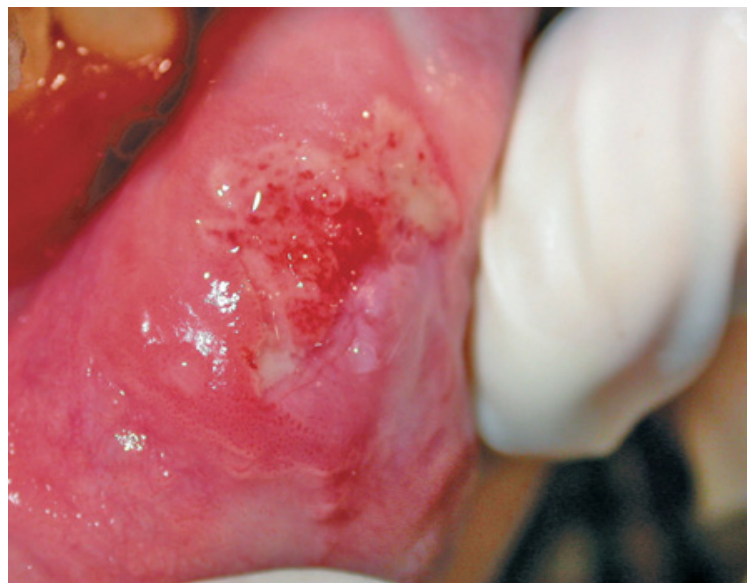

Figure 3 : Oedème autour de l'ulcération labiale inférieure.

Edema around an ulcer situated on the lower lip. 


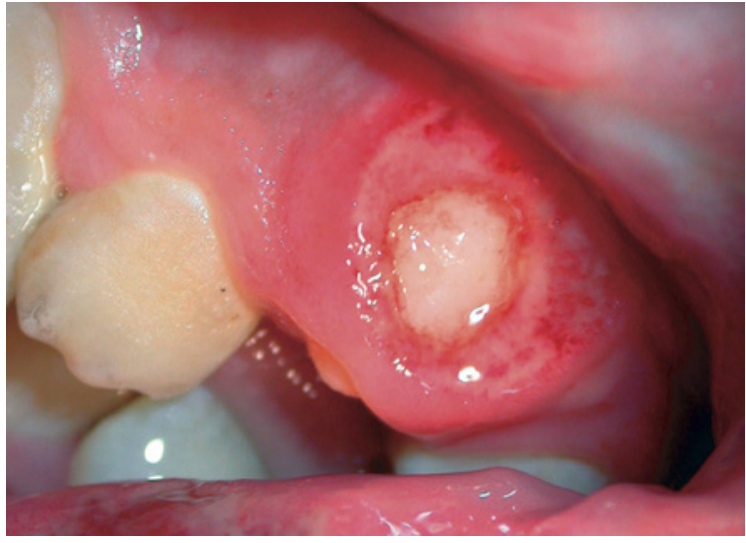

Figure 4 : Ulcération de la gencive attachée en regard du germe de la 23 .

Ulcer on the attached gingiva at the eruption site of 23.

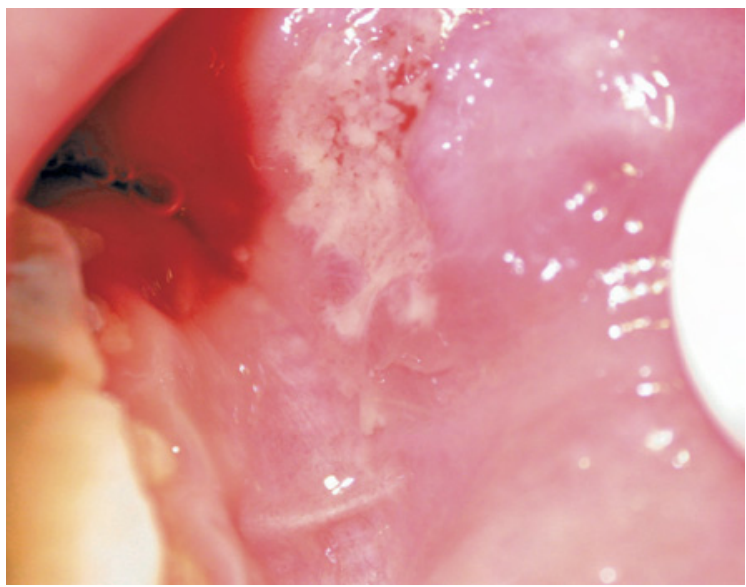

Figure 5 :Ulcération jugale gauche, en regard de la 37, apparue sur un site cicatriciel.

Ulcer on the left buccal mucosa facing 37 which has appeared on a healing site.

fond granuleux, creusant et recouvert par un enduit gris jaunâtre (Fig. 2). Elle était associée à un œdème important qui s'étendait à distance de la lésion, intéressant presque toute la lèvre inférieure (Fig. 3). La lésion a disparu après à un traitement symptomatique local (bains de bouche à base de chlorhéxidine). Trois mois plus tard, on a remarqué l'apparition d'une autre ulcération sur la gencive attachée en regard du germe de la 23, entraînant l'exposition de ce dernier (Fig. 4).

Les investigations de routine ont mis en évidence une anémie ; le bilan hématologique étant normal par ailleurs. La lésion en regard de la canine ne présentait pas histologiquement de caractères spécifiques.
Le traitement par la colchicine, à la dose de $1 \mathrm{mg} / \mathrm{j}$ pendant un mois, a entrainé une guérison complète. Une nouvelle poussée est apparue 6 mois plus tard, sur la joue gauche en regard de la 37, sur un site cicatriciel (Fig. 5).

Après un suivi régulier pendant plus de 2 ans, la confrontation de l'ensemble des éléments recueillis était en faveur de la maladie de Sutton.

\section{DISCUSSION}

L'aphtose buccale regroupe trois formes:

- l'aphtose buccale commune ;

- l'aphtose buccale herpétiforme ;

- l'aphtose buccale majeure, décrite par les auteurs sous plusieurs termes: aphte cicatriciel majeur, stomatite nécrosante, aphtose géante, aphte nécrosant, maladie de Sutton ou périadénite de Sutton ou encore periadenitis mucosa necrotica reccurens ${ }^{[1,2]}$.

L'aphtose buccale majeure représente environ $10 \%$ de l'ensemble des aphtoses buccales. La maladie se distingue par des ulcérations de taille importante, pouvant atteindre, voire dépasser, $3 \mathrm{~cm}$ de diamètre $[3,4,15]$.

Son étiologie reste inconnue. Plusieurs facteurs ont été évoqués : prédisposition génétique et différents autres facteurs, comme une infection bactérienne, l'état psychique, des mécanismes allergiques, le rôle des traumatismes... ${ }^{[4,8]}$. Les lésions présentent une localisation préférentielle pour les zones riches en glandes salivaires accessoires comme les lèvres, la langue, le voile du palais et les piliers antérieurs du voile ${ }^{[4]}$.

Les ulcérations sont larges et profondes, le plus souvent uniques, mais deux ou trois éléments peuvent coexister. La lésion commence par un nodule inflammatoire de la muqueuse qui s'enfonce profondément dans le chorion. Puis il apparait une ulcération est très douloureuse qui présente un contour irrégulier, surélevée par l'œdème. Les formes sévères persistent jusqu'à 6 à 8 semaines, voire plus. Elles débutent souvent à la puberté et ont une évolution chronique qui peut durer une vingtaine d'années ${ }^{[3,5]}$. Les signes fonctionnels sont importants à type de dysphonie, dysphagie, hyper-sialorrhée. La guérison laisse médecine

buccale

chirurgie

buccale

VOL. $14, \mathrm{~N}^{\circ} 1$ 2008

page 43 
médecine

buccale chirurgie buccale

VOL. $14, \mathrm{~N}^{\circ} 1$ 2008

page 44 une cicatrice rétractile qui peut entraîner une gène à l'ouverture buccale ; c'est le cas de notre patient qui présentait une ouverture buccale limitée à deux travers de doigts ayant motivé la consultation [2,6]. Les examens biologiques (NFS) n'ont révélé aucune anomalie hormis une discrète anémie qui ne constitue pas un facteur favorisant de l'aphtose géante. L'aphte géant est plus fréquent chez les patients atteints par le virus de l'immunodéficience acquise (VIH) qui induit un dysfonctionnement de l'immunité résiduelle $[2,6,8]$.

Le diagnostic différentiel doit faire évoquer une infection herpétique, une ulcération traumatique, un carcinome épidermoïde, un lichen plan érosif, un pemphigus vulgaire ou une pemphigoïde cicatricielle [15].

Histologiquement, la maladie de Sutton se caractérise par une lésion inflammatoire de l'épithélium, du tissu conjonctif et des glandes salivaires accessoires, avec des inclusions intranucléaires. Les couches épithéliales sont détruites. L'infiltrat inflammatoire où prédomine les polynucléaires neutrophiles, touchent des vaisseaux de plus gros calibre en profondeur ${ }^{[2,5,8]}$. Pour Ghate et Jorizzo, cités par Steffen [2], la maladie de Sutton se distingue facilement des ulcérations nécrotiques autour des glandes salivaires accessoires.

En général, le traitement des aphtes buccaux nécessite une hygiène buccale stricte [15]. La prise en charge de la maladie de Sutton reste difficile, souvent inefficace, car il n'existe pas de traitement curatif. L'objectif principal est d'assurer une alimentation correcte par le contrôle des symptômes et de réduire la durée d'évolution des lésions et, dans le cas où les ulcérations sont permanentes ou très fréquentes, il doit s'efforcer de diminuer la fréquence des récidives. Le traitement de première intention, local ou général, peut faire appel à des corticoïdes, des antiseptiques, des antibiotiques, des immunomodulateurs ou des anesthésiques locaux [14,15].

Les patients réfractaires à ces traitements peuvent être traités par d'autres molécules administrées par voie générale : dapsone, colchicine, thalidomide, lévamisole, et par d'autres médicaments d'efficacité limitée. Les récidives après l'arrêt du traitement constituent le réel problème de la maladie [15].
Les traitements locaux constituent la première approche thérapeutique des aphtoses buccales récidivantes, mais ils sont rapidement éliminés par frottement ou par rinçage, ce qui diminuent d'autant leur efficacité. Les corticoïdes, en application locale ou en aérosols, représentent le traitement de base de la maladie de Sutton [10,12]. Parmi les molécules utilisées localement, on trouve la triamcinolone acétonide, la béthaméthasone, la fluocinomide. Les corticoïdes peuvent être associés à des adhésifs spécifiques de la muqueuse buccale (orobase) [1,10,13,14]. Les bains de bouche à base de corticoïdes sont indiqués chez les patients qui ne peuvent pas appliquer le produit directement sur les ulcérations ou si la lésion est de grande taille. Revuz, cité par Laccourey [1], suggère l'application des corticoïdes locaux avant la formation de l'ulcération pour être plus efficace. D'autres traitements sont préconisés. Le gluconate chlorhéxidine à $0,2 \%$ réduit le nombre et la durée des ulcérations $[1,12,14]$. Les tétracyclines, en application locale, peuvent réduire la durée de l'évolution et/ou la douleur associée à l'aphtose buccale récidivante. Une étude réalisée par Ylikontiola [11] a montré que l'utilisation de doxymycine cyanoacrylate élimine complètement la douleur en 6 jours et diminue la destruction tissulaire causée par l'ulcération. Certains immunomodulateurs en application topique, ont un effet bénéfique dans le traitement de l'aphtose buccale récidivante : on doit citer l'azelastine, l'interféron alpha 2, la ciclosporine, l'acide 5-amino-salicylique et les prostaglandines E2 [1,12].

Lorsque les traitements locaux ne sont pas efficaces ou lorsque la douleur est violente et/ou les lésions de taille importante ${ }^{[14]}$, le traitement systémique se justifie. La prednisone $(1 \mathrm{mg} / \mathrm{kg} / \mathrm{j})$ est prescrite pendant 1 à 2 semaines. Elle peut être associé à de l'azathioprine ou du cyclophosphamide afin de réduire la dose de corticoïdes $[4,14,15]$. Des études ont montré que la colchicine est également efficace : elle permet de réduire la douleur et la fréquence des récidives. La posologie est de 1,5mg/j pendant 2 mois. La colchicine donne des effets secondaires dont les plus importants sont des troubles gastro-intestinaux et une neutropénie $[6,10,14]$. La thalidomide a une effica- 
cité spectaculaire. La dose d'attaque est de 100 $\mathrm{mg} / \mathrm{jour}$ pendant un à deux mois. La posologie est ensuite réduite progressivement. Sa prescription est limitée par ses deux principaux effets secondaires : la tératogenicité et les neuropathies périphériques ${ }^{[1,10,12-14]}$. D'autres médicaments anti-inflammatoires, immunomodulateurs ou autres peuvent être utilisés comme la dapsone, la ciclosporine ou l'oxypentifylline ${ }^{[9,14]}$.

\section{RÉFÉRENCES}

1 - Laccourrey O, Fadlallah JP, Pages JC, Durand H, Brasnu D, Lowenstein W. Sutton's Disease (Periadenitis Mucosa Necrotica Recurrens). Ann Otol Rhinol Laryngol $1995 ; 104: 301-4$.

2 - Steffen C, Thomas D. The man behind the eponyms: Richard L Sutton: Periadenitis mucosa necrotica recurrens (Sutton' ulcer) and Leucoderma Acquisitum Centrifugum-Sutton's (Halo) Nevus. Am J Dermatopathol 2003 ; 25 : 349-54.

3 - Szpirglas H, Ben Slama L. Aphtes et aphtoses (pp 4954). Pathologie de la muqueuse buccale. Elsevier, Paris, 1999.

4 - Ship JA, Chavez EM, Doerr PA, Hensen BS, Sarmadi M. Reccurens aphthous stomatitis. Quintessence Int $2000 ; 31: 95-108$.

5 - Coulon JP, Piette E. Aphtes banals, aphtose buccale récidivante et maladie de Behçet. Ency Med Chir, Stomatologie, Elsevier, Paris, 22-050-N-10,1999.

6 - Agbo-Godeau S. Aphtoses buccales. Rev Prat 2002 ; 523,78-83.

7 - Ben Slama L. Aphtes et aphtoses. Rev Stomatol Chir Maxillofac 2003 ; 104 : 295-7.

8 - Monteone L. Periadenitis mucosa necrotica reccurens. Oral Surg Oral Med Oral Pathol 1967 ; 23 : 586-91.
En conclusion, il n'existe pas de traitement curatif de la maladie de Sutton capable d'éliminer les récidives. Le cas rapporté a été traité par des antiseptiques, des antibiotiques, des anesthésiques et, en dernier ressort, par la colchicine. II n'y a pas de traitement préventif de la fibrose cicatricielle secondaire aux aphtes géants.

9 - Chandrachekhar J, Liem AA, Cox NH, Paterson AW. Oxypentifylline in the management of recurrent aphthous oral ulcers. Oral Surg Oral Med Oral Pathol Oral Radiol Endod 1999 ; 87 : 564-7.

10 - Jurge S, Kuffer R, Scully C, Porter SR. Mucosal disease series. Recurrent aphthous stomatitis. Oral Dis 2006 ; $12: 1-21$.

11 - Ylikontiola L, Sorsa T, Hayrinen-Immonen R, Salo T. Doxymycine-cyanoacrylate treatment of recurrent aphthous. Oral Surg Oral Med Oral Pathol Oral Radiol Endod 1997 ; 83 : 329-33.

12 - Porter S. Hegarty A, Kaliakatsou F, Hodgson TA, Scully C. Recurrent aphtous stomatitis. Clinics Dermatol 2000 ; $18: 569-78$.

13 - MacPhail L. Topical and systemic therapy for recurrent aphthous stomatitis. Semin Cutan Med Surg1997 ; 16 : 301-7.

14 - Natah SS, Konttinen YT, Enattah NS, Ashammakhi N, Sharkes KA, Häyrinen-Immonen R. Recurrent aphthous ulcers today: a review of the growing knowledge. Int J Oral Maxillofac Surg 2004 ; 33 : 221-34.

15 - Ruhl CM, Gleich LL, Biddinger PW, Gluckman JL. Sutton's disease. Am J Otolaryngol 2003 ; 18 : 214-6. médecine buccale chirurgie buccale

VOL. $14, \mathrm{~N}^{\circ} 1$ 2008

page 45 